\title{
Testing Seismic Models of Hot B Subdwarfs with Gaia Data
}

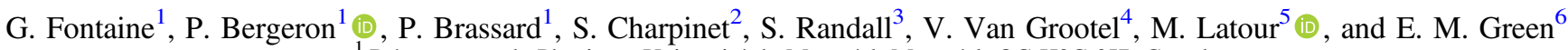 \\ ${ }^{1}$ Département de Physique, Université de Montréal, Montréal, QC H3C 3J7, Canada \\ ${ }^{2}$ CNRS, Université de Toulouse, UPS-OMP, Toulouse, France \\ ${ }^{3}$ ESO, Karl-Schwarzschild-Str. 2, D-85758 Garching bei München, Germany \\ ${ }^{4}$ Space Sciences, Technologies and Astrophysics Research (STAR) Institute, Université de Liège, 19C Allée du 6 Août, B-4000 Liège, Belgium \\ Institute for Astrophysics, Georg-August-University, Friedrich-Hund-Platz 1, D-37077 Göttingen, Germany \\ ${ }^{6}$ Steward Observatory, University of Arizona, 933 North Cherry Avenue, Tucson, AZ 85721, USA \\ Received 2019 May 13; revised 2019 June 6; accepted 2019 June 8; published 2019 July 29
}

\begin{abstract}
We report the results of a study designed to test further the basic validity of the seismic models of hot pulsating B subdwarfs that have been developed over the last two decades. Given estimates of the effective temperature, surface gravity, and mass as obtained from a seismic analysis, and given an estimate of the $\mathrm{He} / \mathrm{H}$ ratio as available from independent spectroscopy, it is possible to compute a "seismic distance" that is suitably corrected for interstellar reddening from a model atmosphere calculation in conjunction with two-band photometry. The test consists of comparing such a distance with that obtained directly from the high-accuracy Gaia parallax measurements that have become available through Data Release 2. Using observed magnitudes in the Gaia $G_{\mathrm{BP}}$, $G_{\mathrm{RP}}$ bandpasses as well as ground-based photometry in the Johnson $B, V$, Strömgren $b, y$, and SDSS $g, r$ filters, we find that all of our seismic estimates fall within $1 \sigma$ of their parallax counterparts. In addition, the derived reddening for our target stars is shown to be generally consistent with Galactic dust reddening and extinction maps. These results apply to a sample of 14 pulsating hot B subdwarfs for which seismic models were published in the past. We also derive useful constraints on the distances to two more pulsators that belong to unresolved binary systems. The excellent agreement found between seismic and parallax distances for the bulk of the sample adds further credibility to the seismic models that have been calculated for pulsators of this type.
\end{abstract}

Key words: stars: atmospheres - stars: oscillations - subdwarfs

\section{Introduction}

Among the many types of pulsating stars currently known, pulsating hot B subdwarf (sdB) stars have turned out to be exceptional asteroseismological laboratories. There are two main families of pulsating sdBs, the short-period $p$-mode pulsators of the V361 Hya type discovered in 1997 (Kilkenny et al. 1997) and the cooler, less compact long-period $g$-mode oscillators of the V1093 Her type discovered in 2003 (Green et al. 2003). A few objects, lying mostly at the boundary between the two instability regions in the surface gravityeffective temperature domain, show both $p$ - and $g$-mode pulsations and are referred to as hybrid pulsators (e.g., Baran et al. 2005; Schuh et al. 2006). These two categories of pulsators show strong analogies with the $\beta$ Cephei/slowly pulsating blue stars on the main sequence (Fontaine et al. 2003). However, sdB stars lie well below the main sequence in the Hertzsprung-Russell diagram on the extreme horizontal branch and are hot $\left(22,000 \mathrm{~K} \lesssim T_{\text {eff }} \lesssim 42,000 \mathrm{~K}\right)$, compact $(5.2 \lesssim \log g \lesssim 6.2)$ core helium burning objects. Their overall properties and evolutionary status were last reviewed by Heber (2016).

Since the discovery that a significant fraction of $\mathrm{sdB}$ stars pulsate, tremendous progress has been made in terms of exploiting their asteroseismological potential (see, e.g., Charpinet et al. 2016 for a recent review). In particular, Brassard et al. (2001) first adapted the forward asteroseismological approach to sdB pulsators using parameterized static models of these stars. In this method, a detailed search in parameter space is carried out with the aim of finding an optimal model whose computed pulsation periods best match the observed periods. The technique was then vastly improved over the years with the development of sophisticated optimization tools and new generations of stellar models. For $p$-mode pulsators, which can be well studied from the ground due to their relatively short periods of a few minutes and their amplitudes of several millimags, asteroseismic studies of the sort have led, typically, to the determination of fundamental parameters such as the total mass, the surface gravity, the effective temperature, and the mass of the H-rich envelope (e.g., Charpinet et al. 2005c; Van Grootel et al. 2013). In some cases inferences about the internal rotation profile could be obtained (e.g., Charpinet et al. 2008). Furthermore, on the basis of such studies, a first view of the empirical mass distribution of $\mathrm{sdB}$ stars was provided by Fontaine et al. (2012).

For their part, the $g$-mode pulsators remained difficult to study from the ground due to their much longer observed periods, from $\sim 0.5$ to $\sim 4 \mathrm{hr}$, and their small amplitudes of typically less than $1 \mathrm{mmag}$ (e.g., Randall et al. 2006b, 2006c). It thus took the advent of space-borne observations to exploit the additional seismic riches associated with $g$-modes, which probe much deeper inside $\mathrm{sdB}$ stars than $p$-modes do. The first attempt to derive the fundamental parameters, as well as to probe the core of an $\mathrm{sdB}$ pulsator, was made by Van Grootel et al. (2010b) for the $g$-mode pulsator KPD 0629-0016 on the basis of CoRoT data (Charpinet et al. 2010). This was followed by the study of Van Grootel et al. (2010a) and Charpinet et al. (2011) on the $g$-mode pulsators KPD $1943+4058$ and KIC 02697388, respectively, using Kepler observations. These investigations led to the strong suggestion that the convective mixed core in sdB stars is significantly larger than expected from standard evolutionary models, thus demonstrating the potential of these stars for investigating the structure and evolution of core helium burning objects. Most recently, 
Charpinet et al. (2019) used Transiting Exoplanet Survey Satellite (TESS) data to model the newly discovered $g$-mode pulsator TIC 278659026, and reached similar conclusions.

To date, a total of 16 pulsating sdB stars $(12 p$-mode and $4 g$ mode variables) have been modeled through the approach pioneered by Brassard et al. (2001). We note that, in most cases, the values of the surface gravity $\log g$ and of the effective temperature $T_{\text {eff }}$ inferred from seismology have been tested against independent measurements obtained through standard quantitative analyses of the time-averaged optical spectrum of a target star. This compatibility with spectroscopy was deemed a necessary condition for searching for, and ultimately accepting, a seismic solution in all but one of these investigations. ${ }^{7}$ It has been an inherent part of the whole approach from the very beginning, and constitutes a fundamental test.

The recent release (DR2) of high-accuracy parallax measurements from Gaia (Gaia Collaboration et al. 2018) provides another opportunity for testing independently the available seismic models for pulsating $\mathrm{sdB}$ stars. Indeed, it is possible to obtain an independent estimate of the distance to a given pulsator by computing a model atmosphere and a synthetic spectrum specified by the seismic values of the surface gravity, effective temperature, and mass (or radius), three of the fundamental properties that routinely come out of such seismic exercises. $^{8}$ This allows for the computation of an absolute magnitude in a given bandpass, which, combined with an observed magnitude, leads to a "seismic distance." This estimate can be refined by taking into account interstellar reddening, since the latter cannot be ignored for these sdB stars typically located at distances of $\sim 1 \mathrm{kpc}$. This requires minimally two-band photometry. The test then consists in comparing the seismic distance with that obtained directly from the Gaia parallax for a given pulsator. A weaker component of the test is to verify if the inferred value of the reddening index, $E(B-V)$, is also compatible with line-of-sight measurements as provided by Galactic dust reddening and extinction maps. The index $E(B-V)$, which measures extinction between Earth and a given star, is deemed acceptable if it is smaller than (or equal to) the total extinction along the line of sight.

In this paper, we consider the 16 pulsating $\mathrm{sdB}$ stars for which detailed seismic models have been derived so far. As is clear from Charpinet et al. (2019) for example, the parameterized forward modeling approach put forward initially by Brassard et al. (2001) has now become a powerful tool for extracting the seismic information contained in the observed pulsation periods of a star. In this rising era of TESS, which is providing tens of new light curves for pulsating sdB stars, the method will find many more applications. In this context, we felt that, with the recent availability of Gaia DR2 data, we could not miss the opportunity to further test it.

\section{Available Data}

\subsection{Basic Properties of the Seismic Models}

Table 1 identifies each of the 16 pulsating sdB stars of interest, along with their seismically determined values of the surface gravity, effective temperature, and total mass as

\footnotetext{
The exception is the binary star PG $0048+091$, for which a seismic model was proposed by Charpinet et al. (2006a) a few years before Østensen et al. (2010) deconvolved the polluted spectrum of the sdB component.

8 Note that, to zeroth order, the atmospheric chemical composition for sdB stars is pure $\mathrm{H}$, but see below.
}

provided in the original analysis papers listed as references. ${ }^{9}$ The quoted uncertainties in the table are the formal errors of the seismic fit in parameter space and do not include possible systematic errors, which remain very difficult to estimate. We note that 4 of the stars listed in Table 1 are $g$-mode pulsators (KPD 1943+4058, KPD 0629-0016, KIC 02697388, and TIC $278659026)$, while the 12 others are $p$-mode pulsators. We note further that in the seismic studies carried out on Feige 48 (Charpinet et al. 2005b), PG 1325+101 (Charpinet et al. 2006b), PG 0911+456 (Randall et al. 2007), and EC 09582 -1137 (Randall et al. 2009), the effective temperature was fixed a priori at the value inferred from spectroscopy. This is because only a few pulsation periods (acting as constraints) were available in those four cases, and fixing the effective temperature provided a welcome additional constraint in searching for a seismic solution in parameter space. The choice of that parameter over others was motivated by the observation that the mechanical structure of a typical sdB star is such that the pulsation periods are much more sensitive, in a relative sense, to a variation of surface gravity than to a variation of the effective temperature (see, e.g., Charpinet 1999). Spectroscopy can provide more precise values of $T_{\text {eff }}$ than asteroseismology for sdB stars, while the converse is true for $\log g$ (Brassard et al. 2001).

It should be pointed out that the target stars chosen in most of the seismic analyses summarized in Table 1 were selected on the basis of their simplicity and stability as pulsators, as well as their brightness. In addition, a conscious effort was made to avoid, if possible, targets with light curves and spectra contaminated by the flux of a companion in unresolved binary systems. In this way, the sample is biased against binarity, which is a frequent phenomenon in sdB stars (see, e.g., Heber 2016). For the specific purpose of seismic modeling, all but one of the stars listed in Table 1 can be considered as isolated. The exception is PG 1336-018, which is a close eclipsing binary system made of a pulsating $\mathrm{sdB}$ component and an M dwarf companion (Kilkenny et al. 1998). This is a spin-orbit synchronized system with a period of $2.4244 \mathrm{hr}$, and is the only case where terms higher than first-order in rotation and tidal interaction had to be taken into account in the seismic modeling itself (see Charpinet et al. 2008). Otherwise, the modeling exercise was carried out within the slow-rotation approximation for isolated stars.

\subsection{Atmospheric Parameters from Quantitative Spectroscopy}

Table 2 gives the atmospheric parameters of the 16 stars of interest as inferred from quantitative spectroscopy, independently of any seismic considerations. These are the surface gravity, the effective temperature, and the number ratio of helium-to-hydrogen in these $\mathrm{H}$-dominated hot stars. The uncertainties given in the table are those quoted in the original references and correspond solely to the formal errors of the spectral fit. In most cases, the atmospheric analyses have been carried out in a homogeneous way, following the method developed by Bergeron et al. (1992) ${ }^{10}$ and using (1) timeaveraged optical spectra gathered by one of us (E.M.G.) at the

\footnotetext{
9 In the case of KIC 02697388, Charpinet et al. (2011) have provided two equally acceptable seismic models, which are very close to each other in parameter space.

${ }^{10}$ This is based on a $\chi^{2}$ minimization procedure that relies on the method of Levenberg-Marquart. Normalized lines of both the observed and model spectra are thus compared, making the method largely free of flux calibration issues.
} 
Table 1

Basic Characteristics of the Seismic Models

\begin{tabular}{|c|c|c|c|c|}
\hline Name & $\begin{array}{c}\log g \\
\left(\mathrm{~cm} \mathrm{~s}^{-2}\right)\end{array}$ & $\begin{array}{l}T_{\text {eff }} \\
(\mathrm{K})\end{array}$ & $\begin{array}{c}M \\
\left(M_{\odot}\right)\end{array}$ & References \\
\hline PG $1047+003$ & $5.800 \pm 0.006$ & $33000 \pm 1600$ & $0.490 \pm 0.014$ & Charpinet et al. (2003) \\
\hline PG 0014+067 & $5.775 \pm 0.009$ & $33940 \pm 3520$ & $0.477 \pm 0.024$ & Charpinet et al. (2005a) \\
\hline PG $1219+534$ & $5.807 \pm 0.006$ & $33640 \pm 1360$ & $0.457 \pm 0.012$ & Charpinet et al. (2005c) \\
\hline Feige 48 & $5.437 \pm 0.006$ & $29580 \pm 370$ & $0.460 \pm 0.008$ & Charpinet et al. (2005b) \\
\hline EC 05217-3914 & $5.730 \pm 0.008$ & $32000 \pm 1800$ & $0.490 \pm 0.020$ & Billères \& Fontaine (2005) \\
\hline PG $1325+101$ & $5.811 \pm 0.004$ & $35050 \pm 220$ & $0.499 \pm 0.011$ & Charpinet et al. (2006b) \\
\hline PG $0911+456$ & $5.777 \pm 0.002$ & $31940 \pm 220$ & $0.390 \pm 0.010$ & Randall et al. (2007) \\
\hline BAL 090100001 & $5.383 \pm 0.004$ & $28000 \pm 1200$ & $0.432 \pm 0.015$ & Van Grootel et al. (2008) \\
\hline EC 09582-1137 & $5.788 \pm 0.004$ & $34805 \pm 230$ & $0.485 \pm 0.011$ & Randall et al. (2009) \\
\hline KPD $1943+4058$ & $5.520 \pm 0.030$ & $28050 \pm 470$ & $0.496 \pm 0.002$ & Van Grootel et al. (2010a) \\
\hline KPD 0629-0016 & $5.450 \pm 0.034$ & $26290 \pm 530$ & $0.471 \pm 0.002$ & Van Grootel et al. (2010b) \\
\hline KIC $02697388^{\mathrm{a}}$ & $5.489 \pm 0.033$ & $25622 \pm 420$ & $0.463 \pm 0.009$ & Charpinet et al. (2011) \\
\hline
\end{tabular}

Notes.

${ }^{\text {a }}$ Seismic Model 1.

${ }^{\mathrm{b}}$ Seismic Model 2.

Table 2

Spectroscopic Constraints

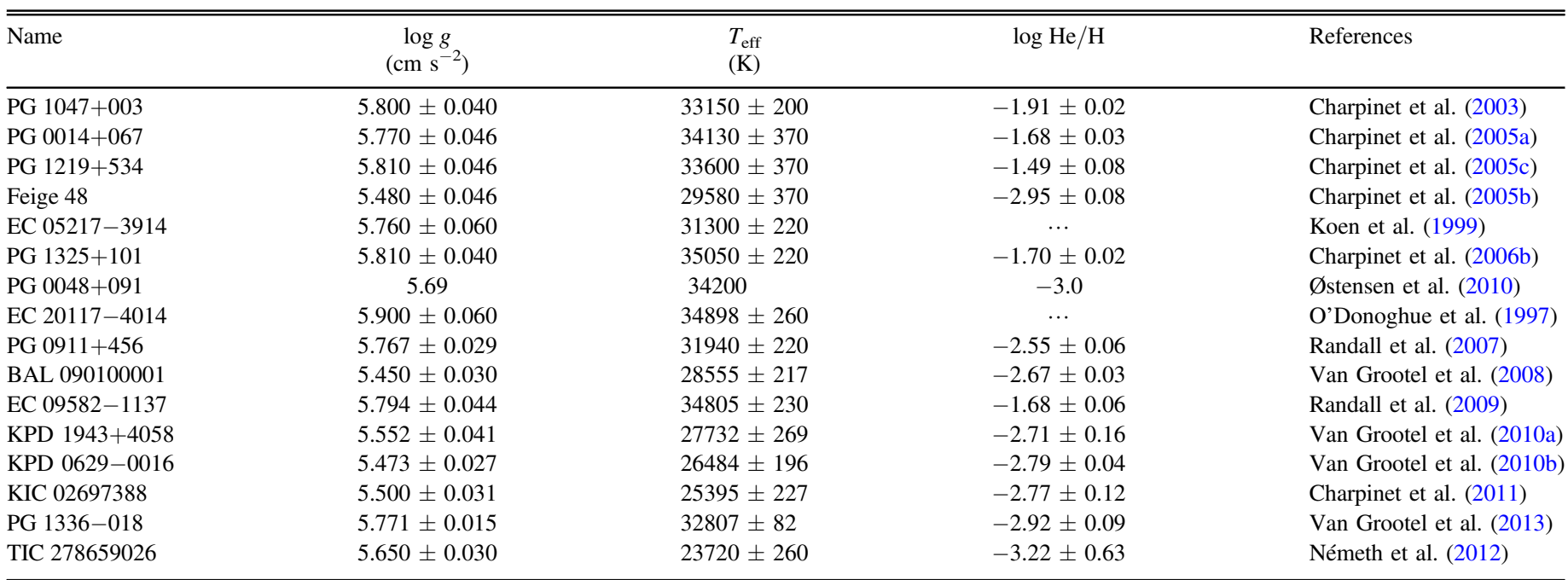

Steward Observatory, and (2) grids of NLTE, metal-blanketed atmosphere models and synthetic spectra computed with the public codes TLUSTY and SYNSPEC at the Universite de Montréal (see, e.g., Brassard et al. 2010). The exceptions are the southern hemisphere objects, EC 05217-3914 and EC 20117-4014, analyzed with pure H models, the binary system PG $0048+091$, and the case of TIC 278659026 analyzed by Németh et al. (2012) using similar tools (TLUSTY/SYNSPEC) as above.

At the beginning of this investigation, four stars in the sample were known to be part of unresolved binary systems. The other targets were not. In descending order in Table 2, Feige 48 was believed, until recently, to consist of a pulsating sdB star and an invisible white dwarf companion (O'Toole et al. 2004). Preliminary work by several of us at the Steward Observatory now indicates that the light curve of the Feige 48 system shows a very weak reflection effect, implying that the companion is rather a late type $M$ dwarf (Latour et al. 2014a). Still, the latter has very little effect on the normalized optical line profiles of $\mathrm{H}$ and $\mathrm{He}$ used in the fitting procedure employed to derive the atmospheric parameters of the $\mathrm{sdB}$ component (see, e.g., Latour et al. 2014b). In contrast, the optical spectrum of PG $0048+091$ is significantly polluted by the light of a G0G2 main-sequence companion according to Koen et al. (2004). The amount of pollution is even higher in the EC 20117-4014 system due to the presence of an F5 dwarf star (O'Donoghue et al. 1997). In both cases, deconvolution procedures were needed to infer the atmospheric parameters of the pulsating sdB component. As a consequence, those estimates are more uncertain (Østensen et al. 2010). Finally, PG 1336-018 has also a much fainter M5 dwarf companion, according to Kilkenny et al. (1998), so the light pollution issue is much 
reduced in this case, despite a significant reflection effect. Therefore, fitting its $\mathrm{H}$ and $\mathrm{He}$ spectral lines shortward of $\sim 5000 \AA$ gave reliable values of its atmospheric parameters (Charpinet et al. 2008).

\subsection{Photometric Data}

Table 3 provides a compilation of the available two-band photometry that we found in the literature. We used the Gaia Archive DR2 to extract, for each star of interest, the apparent magnitude $G_{\mathrm{BP}}$ (second column) and color index $\left(G_{\mathrm{BP}}-G_{\mathrm{RP}}\right)$ (third column). This photometry from Gaia is both very accurate and homogeneous. Although generally less accurate, very good ground-based data exist for many of these relatively bright stars, and we found it interesting to investigate how the results would fare against each other. Hence, we extracted useful data for 13 stars in the standard Johnson system, $V$ and $(B-V)$ (columns 4 and 5), for 8 stars in the Strömgren system, $y$ and $(b-y)$ (columns 6 and 7), and 4 stars in the SDSS ugriz system, $g$ and $(g-r)$ (columns 8 and 9). Altogether, we compiled magnitudes and color indices for 41 pairs.

Among other features, using the $\left(G_{\mathrm{BP}}-G_{\mathrm{RP}}\right)$ index, we draw attention to the very red color measured in the binary systems PG 0048+091 and EC 20117-4014 compared to single stars of similar effective temperature. Of course, there is no surprise here, as both objects contain a main-sequence companion that contributes significantly to (in the case of the G0-G2 companion in PG 0048+09), and even dominates (in the case of the F5 companion in EC 20117-4014), the luminosity of the system in the optical domain as indicated above. Also, the $\left(G_{\mathrm{BP}}-G_{\mathrm{RP}}\right)$ index of KPD $0629-0016$ is quite red, although not as extreme in comparison, but, in this case, interstellar reddening is surely the culprit, as the star is not known to be binary and lies in the galactic plane.

For PG 0048+091 and EC 20117-4014 the optical colors appear to be hopelessly too contaminated to be useful, but additional information can be gathered about them. Indeed, O'Donoghue et al. (1997) estimated from their deconvolution process that the apparent visual magnitude of the $\mathrm{sdB}$ component in EC 20117-4014 is $V=13.55 \pm 0.05$ (compared to a magnitude of $V=12.47 \pm 0.01$ for the system). Combined with a seismic estimate of the absolute magnitude of the pulsator in the $V$ band, $M_{V}=4.48 \pm 0.14$, obtained as described below, allows us to derive an upper limit (uncorrected for interstellar reddening) on the seismic distance. In the case of PG 0048+091, we assume that the companion has a representative absolute magnitude of $M_{V}=4.9$ for a G1V star. Coupled to a seismic value of $M_{V}=4.40 \pm 0.12$ for the sdB component, this leads to an estimate of its apparent magnitude of $V \simeq 14.8$ (compared to a magnitude of $V=14.26 \pm 0.02$ for the system). From those, an upper limit on the seismic distance can again be derived, as is done below.

We also searched the Two Micron All Sky Survey (2MASS) catalog, mostly through the $(J-H)$ index, for the IR signature of potential companions in all of our 16 target stars and, in particular, in the 12 objects not known to be binaries (and see Green et al. 2008). In this way, we uncovered an IR excess in PG 0014+067, highly suggestive of the presence of a cool companion. According to Østensen et al. (2010), the orbital period would have to be longer than a few days for this system (radial velocity variations have been searched for, but only for periods of days and less), but this is also the case for PG 0048 +091 (the actual orbital period is not known, but is much

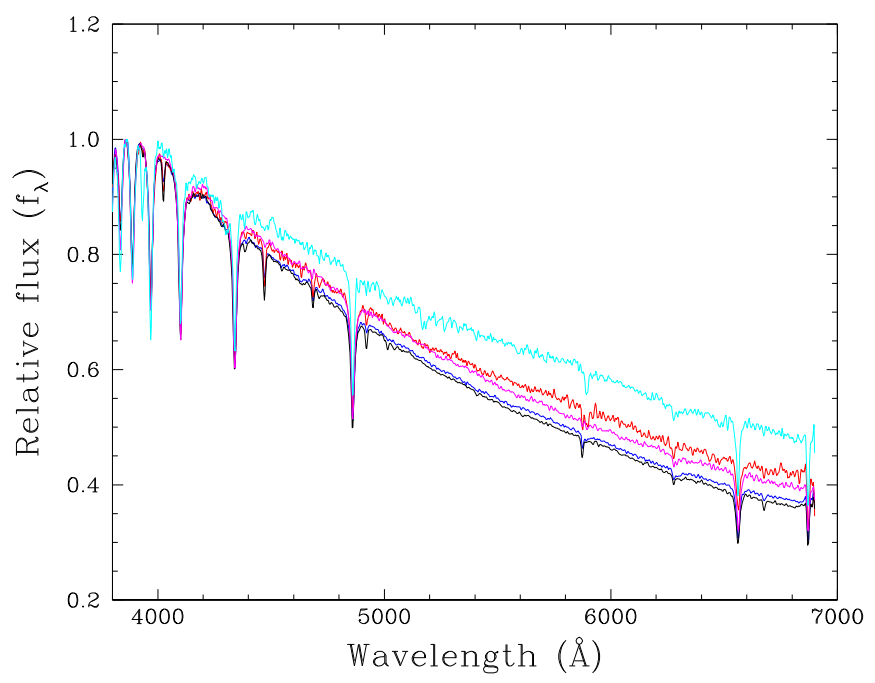

Figure 1. Comparison of the flux-calibrated, low-resolution spectra of PG 0048 +091 (cyan), PG 0014+067 (red), PG 1336-018 (magenta), PG 1047+003 (blue), and PG $1219+534$ (black). These data have been obtained with the Boller and Chivens Spectrograph attached to the Bok Telescope of the Steward Observatory. The claimed accuracy of the flux calibration is a few percent. These five pulsators all have similar estimates of $T_{\text {eff }}$ and $\log g$ (see Table 2). PG $1047+003$ and PG $1219+534$ show no trace of binarity and can be considered as isolated stars. PG $0048+091$ is known to be an unresolved binary containing an sdB and a G1 dwarf companion. Likewise, PG 1336-018 is also an unresolved binary, but contains a much fainter M5 dwarf companion displaying a strong reflection effect. Very likely, PG 0014+067 is also an unresolved binary system that contains a somewhat earlier dwarf than M5.

longer than several days according to Reed et al. 2019) and EC 20117-4014 (the orbital period has recently been determined to be 792.3 days by Otani et al. 2018).

In this context, we compare, in Figure 1, the carefully fluxcalibrated spectra of five objects of interest, all with similar values of the effective temperature of the $\mathrm{sdB}$ component as given in Table 2. Our aim here is to illustrate the slope of these spectra, an exercise that should ideally be carried out for stars with the same values of $\log g$ and, particularly, $T_{\text {eff }}$. We picked two reference stars, PG $1047+003\left(T_{\text {eff }}=33,150 \mathrm{~K}\right)$ and PG $1219+534\left(T_{\text {eff }}=33,600 \mathrm{~K}\right)$, that can be considered as single as they show no IR excess and no radial velocity variations, at least on timescales of hours to days. We also picked the known binaries PG 1336-018 ( $T_{\text {eff }}=32,807 \mathrm{~K}$; M5V companion $)$ and PG 0048+091 ( $T_{\text {eff }}=34,200 \mathrm{~K}$; G1V companion) to compare with the potential new binary PG $0014+067\left(T_{\text {eff }}=34,130 \mathrm{~K}\right)$. We do not have a similar spectrum for the southern hemisphere binary star EC $20117-4014$ ( $T_{\text {eff }}=34,898 \mathrm{~K}$; F5V companion), and, at $T_{\text {eff }}=29,580 \mathrm{~K}$, Feige 48 is too cool for proper comparison. What Figure 1 clearly shows is that the optical spectrum of PG 0014+067 is indeed polluted by the light of a companion, likely somewhat earlier than the M5 dwarf in the comparison system PG 1336-018. To our knowledge, this is the first time PG 0014+067 has revealed itself as an unresolved binary system. We thus retain 5 unresolved binary stars in our sample of 16 pulsators, and we anticipate consequences in our discussion below of interstellar versus intrinsic reddening.

\section{Method and Results}

\subsection{Model Absolute Magnitudes and Colors}

We started with a model atmosphere grid for sdB stars initially developed by Brassard et al. (2010). The grid covers the ranges of $20,000 \leqslant T_{\text {eff }} \leqslant 50,000 \mathrm{~K}$ in steps of $2000 \mathrm{~K}$, 
Table 3

Available Photometry

\begin{tabular}{|c|c|c|c|c|c|c|c|c|}
\hline Name & $G_{\mathrm{BP}}$ & $\left(G_{\mathrm{BP}}-G_{\mathrm{RP}}\right)$ & $V$ & $(B-V)$ & $y$ & $(b-y)$ & $g$ & $(g-r)$ \\
\hline PG 1047+003 & $13.213 \pm 0.007$ (a) & $-0.518 \pm 0.007$ & $13.474 \pm 0.004(\mathrm{~b})$ & $-0.290 \pm 0.003$ & $13.484 \pm 0.003(\mathrm{c})$ & $-0.133 \pm 0.004$ & $\cdots$ & $\cdots$ \\
\hline PG 0014+067 & $15.887 \pm 0.007$ (a) & $-0.348 \pm 0.008$ & $16.089 \pm 0.022(\mathrm{~d})$ & $-0.197 \pm 0.021$ & $\cdots$ & $\cdots$ & $15.837 \pm 0.004(\mathrm{e})$ & $-0.452 \pm 0.006$ \\
\hline PG $1219+534$ & $12.936 \pm 0.006$ (a) & $-0.548 \pm 0.006$ & $\ldots$ & $\ldots$ & $13.235 \pm 0.029(\mathrm{c})$ & $-0.157 \pm 0.017$ & $\ldots$ & $\ldots$ \\
\hline Feige 48 & $13.235 \pm 0.009$ (a) & $-0.438 \pm 0.010$ & $13.480 \pm 0.026$ (f) & $-0.250 \pm 0.013$ & $13.446 \pm 0.029(\mathrm{c})$ & $-0.116 \pm 0.017$ & $\ldots$ & $\ldots$ \\
\hline EC 05217-3914 & $15.296 \pm 0.006$ (a) & $-0.490 \pm 0.007$ & $15.570 \pm 0.020(\mathrm{~g})$ & $-0.280 \pm 0.020$ & $15.550 \pm 0.020(\mathrm{c})$ & $-0.110 \pm 0.020$ & $\ldots$ & $\cdots$ \\
\hline PG $1325+102$ & $13.726 \pm 0.008$ (a) & $-0.539 \pm 0.009$ & $14.019 \pm 0.036(\mathrm{~d})$ & $-0.285 \pm 0.042$ & $14.019 \pm 0.012(\mathrm{c})$ & $-0.134 \pm 0.002$ & $13.710 \pm 0.030(\mathrm{e})$ & $-0.550 \pm 0.036$ \\
\hline PG 0048+091 & $14.242 \pm 0.005$ (a) & $0.230 \pm 0.004$ & $14.261 \pm 0.020(\mathrm{~h})$ & $0.193 \pm 0.050$ & $\ldots$ & $\ldots$ & $\ldots$ & $\ldots$ \\
\hline EC 20117-4014 & $12.406 \pm 0.007$ (a) & $0.248 \pm 0.007$ & $12.470 \pm 0.010$ (i) & $0.130 \pm 0.010$ & $\cdots$ & $\cdots$ & $\cdots$ & $\cdots$ \\
\hline PG 0911+456 & $14.435 \pm 0.008$ (a) & $-0.521 \pm 0.009$ & $14.670 \pm 0.022(\mathrm{~d})$ & $-0.259 \pm 0.031$ & $\ldots$ & $\cdots$ & $14.378 \pm 0.003(\mathrm{e})$ & $-0.521 \pm 0.004$ \\
\hline BAL 090100001 & $11.929 \pm 0.007$ (a) & $-0.361 \pm 0.009$ & $12.100 \pm 0.100(\mathrm{j})$ & $-0.240 \pm 0.100$ & $\cdots$ & $\cdots$ & $\ldots$ & $\ldots$ \\
\hline EC 09582-1137 & $15.017 \pm 0.009$ (a) & $-0.458 \pm 0.009$ & $15.260 \pm 0.050(\mathrm{k})$ & $-0.250 \pm 0.030$ & $\cdots$ & $\cdots$ & $\cdots$ & $\ldots$ \\
\hline KPD $1943+4058$ & $14.752 \pm 0.007$ (a) & $-0.320 \pm 0.007$ & $14.866 \pm 0.027(1)$ & $-0.147 \pm 0.017$ & $14.906 \pm 0.049$ (c) & $-0.024 \pm 0.037$ & $\cdots$ & $\ldots$ \\
\hline KPD 0629-0016 & $14.857 \pm 0.007$ (a) & $-0.073 \pm 0.008$ & $\ldots$ & $\ldots$ & $15.014 \pm 0.024(\mathrm{c})$ & $0.017 \pm 0.023$ & $\cdots$ & $\cdots$ \\
\hline KIC 02697388 & $15.127 \pm 0.004$ (a) & $-0.274 \pm 0.005$ & $15.234 \pm 0.020(\mathrm{~d})$ & $-0.164 \pm 0.030$ & $\cdots$ & $\cdots$ & $15.003 \pm 0.003(\mathrm{e})$ & $-0.416 \pm 0.004$ \\
\hline PG 1336-018 & $13.206 \pm 0.019$ (a) & $-0.424 \pm 0.024$ & $\cdots$ & $\cdots$ & $13.450 \pm 0.093(\mathrm{c})$ & $-0.107 \pm 0.009$ & $\cdots$ & $\cdots$ \\
\hline TIC 278659026 & $11.433 \pm 0.008$ (a) & $-0.398 \pm 0.009$ & $11.570 \pm 0.090(\mathrm{~m})$ & $-0.220 \pm 0.100$ & $\cdots$ & $\cdots$ & $\cdots$ & $\cdots$ \\
\hline
\end{tabular}

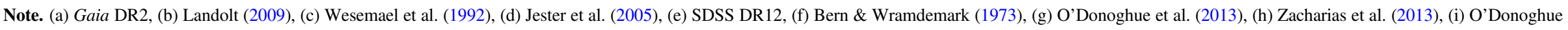
et al. (1997), (j) Van Grootel (2008), (k) Kilkenny et al. (2006), (l) Allard et al. (1994), (m) Hog et al. (2000). 
$4.6 \leqslant \log g \leqslant 6.4$ in steps of $0.2 \mathrm{dex}$, and $-4.0 \leqslant \log \mathrm{He} / \mathrm{H} \leqslant$ 0.0 in steps of 0.5 dex. These are NLTE model atmospheres computed with TLUSTY that include metal line blanketing using a representative metallicity $(\mathrm{C}=0.1, \mathrm{~N}=1.0, \mathrm{O}=0.1$, $\mathrm{S}=1.0, \mathrm{Si}=0.2, \mathrm{Fe}=1.0 \times$ solar values). For each of these models, a synthetic spectrum was computed using SYNSPEC. The flux was then convolved with a bandpass. If the radius is known, this can be converted into an absolute magnitude for that bandpass. In a two-band system, a theoretical color index is then obtained by taking the difference between the two absolute magnitudes.

As given in Table 1, a typical seismic analysis provides, among other properties, estimates of the effective temperature, surface gravity, and total mass (and, hence, of the radius) of a pulsator. It does not constrain the atmospheric composition, in particular the helium-to-hydrogen ratio. However, we note that the absolute magnitudes and colors for these sdB models in the broad bandpasses of interest depend only slightly on the values of $\log \mathrm{He} / \mathrm{H}$, especially for the subsolar values encountered in sdB stars (and see Table 2). In other words, we could have used pure $\mathrm{H}$ models to compute our absolute magnitudes. Still, to be somewhat more accurate, we adopted the values of $\log \mathrm{He} / \mathrm{H}$ given in Table 2 for a given pulsator and used -2.0 in the two cases where spectroscopy has not provided an estimate.

Given a radius, we estimated the absolute magnitude for a given bandpass in 3D space $\left(T_{\text {eff }}, \log g, \log \mathrm{He} / \mathrm{H}\right)$ using parabolic interpolation. This has the advantage of leading naturally to an estimate of the uncertainty on the derived absolute magnitude given the uncertainties on the three basic parameters. A comparison with the apparent magnitude provides, of course, an estimate of the distance. A comparison of the theoretical color index with the observed value provides, in conjunction with an extinction model, a measure of the interstellar reddening. This leads to a revised estimate of the distance, corrected for reddening.

\subsection{Distance and Reddening Index}

The distance $d$ to a star is derived through the standard equation

$$
\log d=\frac{a-A_{a}-M_{a}+5}{5},
$$

where $d$ is in parsec, $a$ is the apparent magnitude, $A_{a}$ is the absorption coefficient, and $M_{a}$ is the absolute magnitude in a given photometric bandpass $(a)$. The absorption coefficient is related to the usual interstellar reddening index $E(B-V)$ through the relation

$$
A_{a}=K_{a} E(B-V),
$$

where $K_{a}$ is a bandpass-dependent quantity that can be evaluated from a model of the interstellar extinction. We took the values of $K_{a}$ from the work of Schlafly \& Finkbeiner (2011) for the bandpasses of interest here (see column 4 in their Table 6). These are $B$ and $V$ (Landolt), $b$ and $y$ (Strömgren), and $g$ and $r$ (SDSS). In addition, using again the work of Schlafly \& Finkbeiner (2011), we estimated the corresponding coefficient for the Gaia bandpasses $G_{\mathrm{BP}}$ (effective wavelength of $5320 \AA$ ) and $G_{\mathrm{RP}}(7970 \AA)$ as $K_{\mathrm{BP}}=2.81$ and $K_{\mathrm{RP}}=1.56$.

The standard reddening index $E(B-V)$ can be estimated from the inferred reddening index in a two-band system ( $a$ and $b$ )

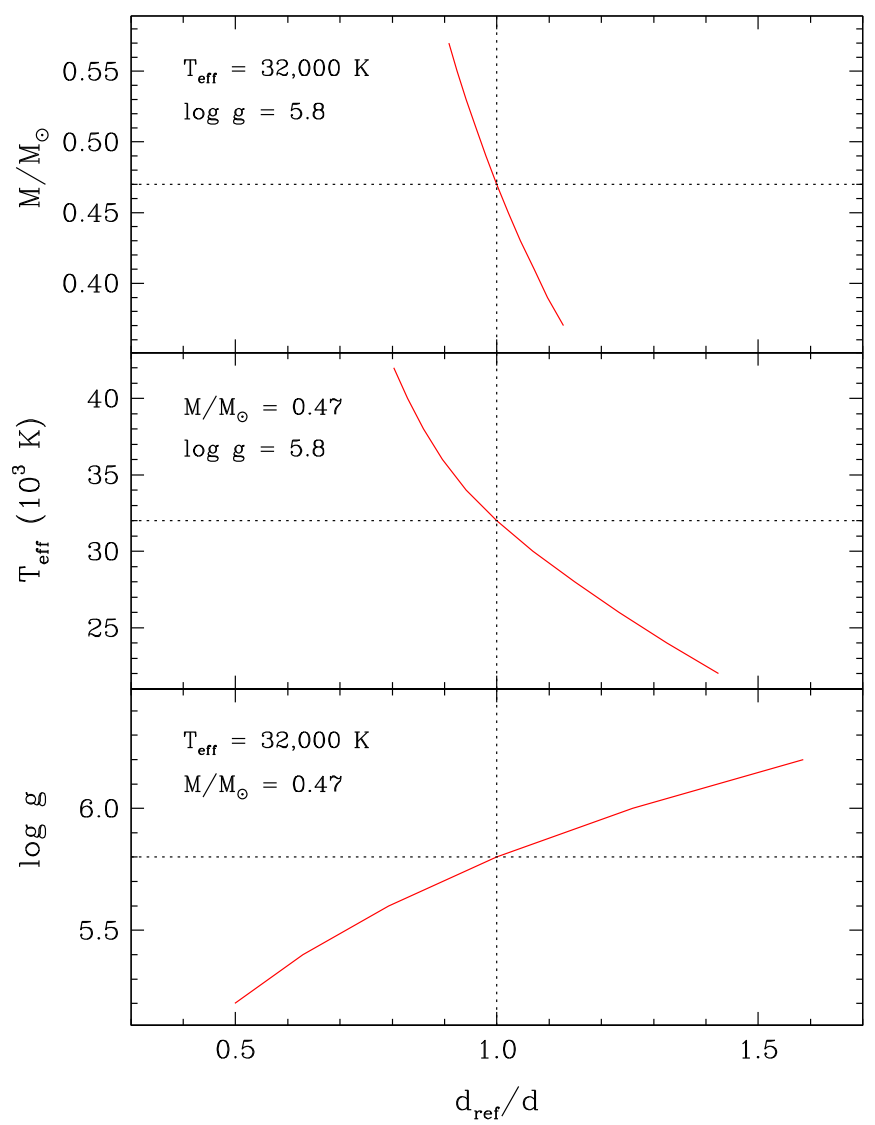

Figure 2. Variations of the inferred distance with respect to a reference model obtained by changing one seismic parameter in the range of interest for $\mathrm{sdB}$ stars, while keeping the other two fixed.

from the equation

$$
E(B-V)=\frac{E(a-b)}{\left(K_{a}-K_{b}\right)},
$$

in conjunction with

$$
E(a-b)=(a-b)-(a-b)^{0},
$$

where $(a-b)$ is the measured color index, and $(a-b)^{0}$ is the theoretical (unreddened) color index. The uncertainty in the derived distance to a pulsator is estimated, taking into account the uncertainties on the absolute magnitude $M_{a}$ and theoretical color index $(a-b)^{0}$ (from parabolic interpolation in 3D space in our grids of models as well as a contribution from the uncertainty on the seismic radius), and the photometric errors on the observed magnitudes $a$ and $b$. These errors are added in quadrature.

\subsection{Distance and Seismic Parameters}

In the current test, seismology provides input values of the effective temperature, surface gravity, and total mass. These are used to compute the absolute magnitude in a given bandpass in conjunction with an estimate of the helium-to-hydrogen number ratio taken from spectroscopy. The effects of the three seismic parameters on the distance are correlated, but it is still instructive to examine the effects of varying individually each of these parameters. This is best done by computing a difference of distance with respect to a reference model. From 
Table 4

Comparison of Distances

\begin{tabular}{|c|c|c|c|c|c|}
\hline Name & $\begin{array}{c}d(\text { Gaia }) \\
\quad(\mathrm{pc})\end{array}$ & $\begin{array}{c}d\left(G_{\mathrm{BP}} G_{\mathrm{RP}}\right) \\
(\mathrm{pc})\end{array}$ & $\begin{array}{c}d(B V) \\
(\mathrm{pc})\end{array}$ & $\begin{array}{c}d(b y) \\
(\mathrm{pc})\end{array}$ & $\begin{array}{c}d(g r) \\
(\mathrm{pc})\end{array}$ \\
\hline PG $1047+003$ & $687 \pm 37$ & $609 \pm 49$ & $609 \pm 42$ & $613 \pm 39$ & $\ldots$ \\
\hline PG 0014+067 & $2794 \pm 1037$ & $1812 \pm 277$ & $1840 \pm 306$ & $\ldots$ & $1933 \pm 268$ \\
\hline PG $1219+534$ & $549 \pm 14$ & $532 \pm 35$ & $\ldots$ & $534 \pm 48$ & $\ldots$ \\
\hline Feige 48 & $822 \pm 27$ & $769 \pm 28$ & $777 \pm 40$ & $784 \pm 50$ & $\ldots$ \\
\hline EC 05217-3914 & $1590 \pm 108$ & $1636 \pm 154$ & $1622 \pm 86$ & $1603 \pm 182$ & $\ldots$ \\
\hline PG $1325+102$ & $862 \pm 51$ & $820 \pm 23$ & $808 \pm 74$ & $823 \pm 19$ & $827 \pm 68$ \\
\hline PG 0048+091 & $1058 \pm 48$ & $\ldots$ & $1197 \pm 145^{\mathrm{a}}$ & $\ldots$ & $\ldots$ \\
\hline EC 20117-4014 & $587 \pm 13$ & $\ldots$ & $653 \pm 58^{\mathrm{a}}$ & $\cdots$ & $\ldots$ \\
\hline PG $0911+456$ & $1035 \pm 75$ & $958 \pm 27$ & $903 \pm 63$ & $\ldots$ & $941 \pm 21$ \\
\hline BAL 090100001 & $365.6 \pm 8.6$ & $386 \pm 31$ & $401 \pm 100$ & $\ldots$ & $\ldots$ \\
\hline EC 09582-1137 & $1553 \pm 139$ & $1376 \pm 40$ & $1372 \pm 112$ & $\cdots$ & $\cdots$ \\
\hline KPD $1943+4058$ & $1274 \pm 51$ & $1242 \pm 73$ & $1152 \pm 93$ & $1159 \pm 155$ & $\cdots$ \\
\hline KPD 0629-0016 & $1011 \pm 50$ & $1022 \pm 67$ & $\ldots$ & $1134 \pm 116$ & $\ldots$ \\
\hline KIC 02697388 & $1262 \pm 64$ & $1328 \pm 76$ & $1319 \pm 133$ & $\ldots$ & $1366 \pm 76$ \\
\hline KIC $02697388^{c}$ & $1262 \pm 64$ & $1295 \pm 79$ & $1288 \pm 133$ & $\ldots$ & $1332 \pm 78$ \\
\hline PG 1336-018 & $552 \pm 19$ & $541 \pm 26$ & $\ldots$ & $564 \pm 41$ & $\ldots$ \\
\hline TIC 278659026 & $203.7 \pm 2.1$ & $221 \pm 21$ & $210 \pm 56$ & $\ldots$ & $\cdots$ \\
\hline
\end{tabular}

Notes.

a Distance not corrected for interstellar reddening.

b Seismic Model 1 from Charpinet et al. (2011).

c Seismic Model 2 from Charpinet et al. (2011).

Equation (1), one can compute a difference of distance

$$
\log d-\log d_{\mathrm{ref}}=\frac{M_{\mathrm{ref}}-M}{5},
$$

which depends only on the absolute magnitude and is valid for any bandpass.

We considered a representative $\mathrm{sdB}$ model specified by the values of $M / M_{\odot}=0.47, T_{\text {eff }}=32,000 \mathrm{~K}$, and $\log g=5.8$ (and $\log \mathrm{He} / \mathrm{H}=-2.0)$. We next computed the ratio $d_{\text {ref }} / d$ by varying successively one of the seismic parameters, keeping the other two fixed. The results are displayed in Figure 2, which shows how the distance depends on each of the parameters in the ranges of interest for sdB stars. That is, the ranges of $22,000 \lesssim$ $T_{\text {eff }} \lesssim 42,000 \mathrm{~K}, 5.2 \lesssim \log g \lesssim 6.2$, and $0.37 \lesssim M / M_{\odot} \lesssim 0.57$ (see, e.g., Heber 2016). Changing the mass by $\pm 0.1 M_{\odot}$ about $0.47 M_{\odot}$ implies a variation of about $\pm 10 \%$ in distance, while changing the surface gravity by \pm 0.5 dex about $\log g=5.7$ implies a variation in distance some five times larger.

\subsection{Results}

The main results of this study are summarized in Table 4 , where the Gaia distance (the inverse of the trigonometric parallax) for each pulsator is compared with seismic estimates derived from the method described above. Altogether, we obtained 40 values and 2 upper limits on the distance using seismic data and 4 different sources of photometry. Of central interest and significance here, all of these values overlap within $1 \sigma$ with the parallax distances. We thus conclude that, within these uncertainties, all of the seismic models are compatible with Gaia parallaxes. They pass the test proposed at the outset.

The seismic distances are generally determined more precisely on the basis of Gaia photometry than in other photometric systems. This is a consequence of the superior quality of those photometric measurements compared to ground-based data (see Table 3). Also, the derived uncertainties on the seismic distances, while generally larger than those on the Gaia distances, become comparable in several cases. Given that the seismic and parallax distances overlap within $1 \sigma$, we do not observe systematic effects either with the distance itself or with colors. We illustrate this with the four plots Figures 3(a) through (d). Note that these plots do not include PG 0014+067, which falls offscale with a Gaia distance of $d=2794 \pm 1037$ pc. This is the farthest and faintest star in our sample, and is characterized by an atypically large uncertainty in its parallax measurement. We come back to that point below.

Our method also provides estimates of interstellar reddening, and this can be used to further test our results, although more loosely than in the case of the distance. The derived reddening index, $E(B-V)$, is listed in Table 5, to be compared with lineof-sight measurements, $E(B-V)_{\text {mean }}$, as given by Galactic dust reddening and extinction maps and using the Schlafly \& Finkbeiner (2011) values. ${ }^{11}$ To pass the test, the derived reddening index, which measures the extinction between Earth and the star, should be smaller than (or equal to) the total extinction along the line of sight measured by $E(B-V)_{\text {mean }}$. Table 5 indicates that this is indeed the case for the majority of our targets, except for three stars, PG 0014+067, Feige 48, and PG 1336-018, especially on the basis of Gaia photometry.

Given that these three objects are binary stars likely accompanied by faint main-sequence $\mathrm{M}$ stars, the most logical explanation for this "failure" is that the available photometry is sensitive enough to have picked intrinsic reddening along with interstellar reddening in these systems. There are hints that this is indeed the case; the $E(B-V)$ index inferred from $G_{\mathrm{BP}} G_{\mathrm{RP}}$ photometry is larger than those inferred from $B V, b y$, and $g r$ data (with caution due to the uncertainties). This behavior is what is expected in the presence of a faint red companion, given that the effective wavelength coverage in the $G_{\mathrm{BP}} G_{\mathrm{RP}}$

\footnotetext{
${ }^{11}$ No determination of interstellar reddening was possible on the basis of the polluted colors of the binary systems PG 0048+091 and EC 20117-4014.
} 


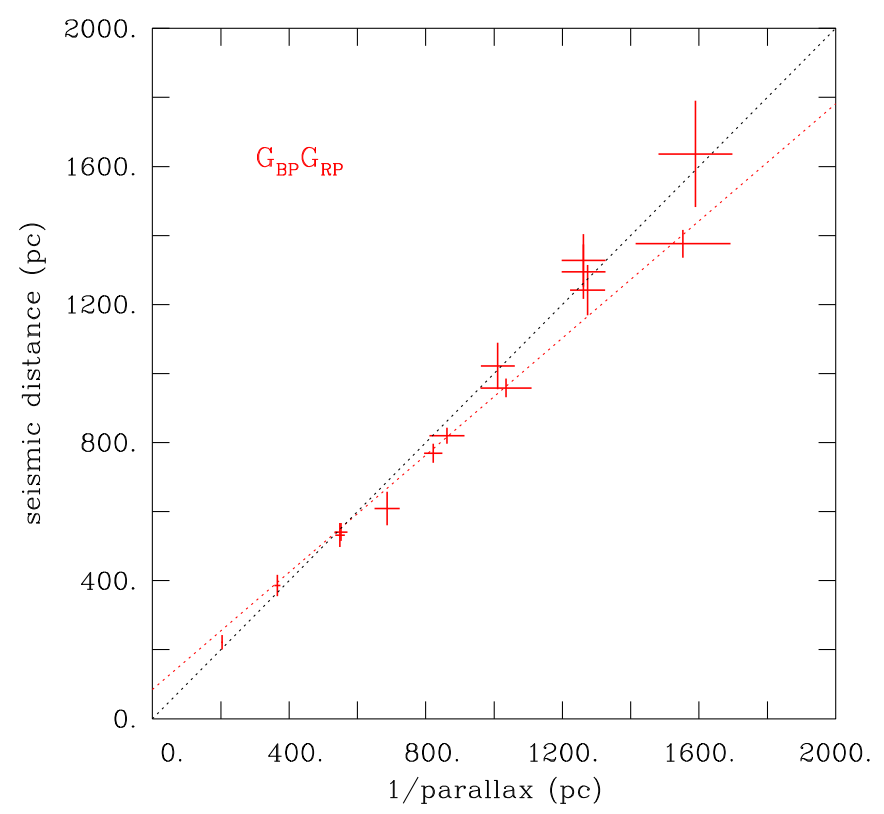

(a)

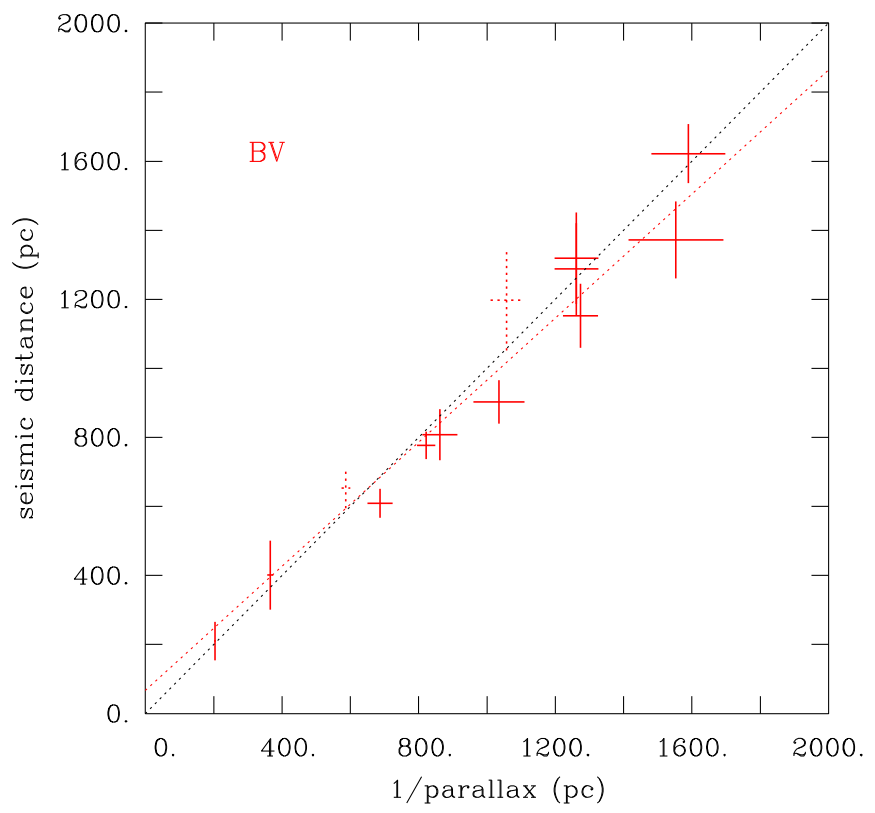

(b)

Figure 3. (a) Comparison of the Gaia distance (obtained by simply inverting the measured parallax) with the distance derived by combining a seismic model with a model atmosphere and measured Gaia magnitudes in the two bands $G_{\mathrm{BP}}$ and $G_{\mathrm{RP}}$. The latter is corrected for the effects of interstellar reddening. The plot does not include PG 0014+067, which is an outlier due to the uncertainty in the Gaia distance of $d=2794 \pm 1037 \mathrm{pc}$ (see the text). The black dotted line shows the 1:1 correspondence, while the red dotted line is a formal $\chi^{2}$ fit giving a correlation coefficient of 0.984. (b) Similar to Figure 3(a), but based on the Johnson magnitudes $B$ and $V$. The correlation coefficient is equal to 0.972 . The seismic distances to two binary stars, PG 0048+091 and EC 20117-4014, are upper limits obtained under the assumption of no interstellar reddening. They are indicated by dotted crosses. (c) Similar to Figure 3(a), but based on the Strömgren magnitudes $b$ and $y$. The correlation coefficient is equal to 0.980. (d) Similar to Figure 3(a), but based on the SDSS magnitudes $g$ and $r$. The correlation coefficient is equal to 0.881 .

system, 5320-7970 $\AA$, is "redder" than those in the other systems (4329-5421 $\AA$ in $B V, 4671-5479 \AA$ in $b y$, and 4717 $-6165 \AA$ in $g r$, and therefore more sensitive to such a presence.

By construction, all of the reddening inferred in our approach is assumed to be due to interstellar absorption. In the case of the three binary systems of interest, this implies that too much reddening (sum of intrinsic and interstellar) has been used, meaning that the derived seismic distances in Table 4for those three objects-are underestimated. Disregarding the formal uncertainties for a moment, the seismic distances for PG 0014+067 and Feige 48 are indeed shorter than the parallax values. This prompted us to reassess our estimates of seismic distances for these three objects, given that we cannot infer interstellar reddening due to the presence of a red companion.

On the one hand, one can obtain an estimate of the upper limit on the seismic distance by neglecting reddening completely (as was done in Table 4 for PG $0048+091$ and EC 20117-4014). The results are reported as $d(\max )$ in Table 6, to be compared again with the Gaia values. On the 


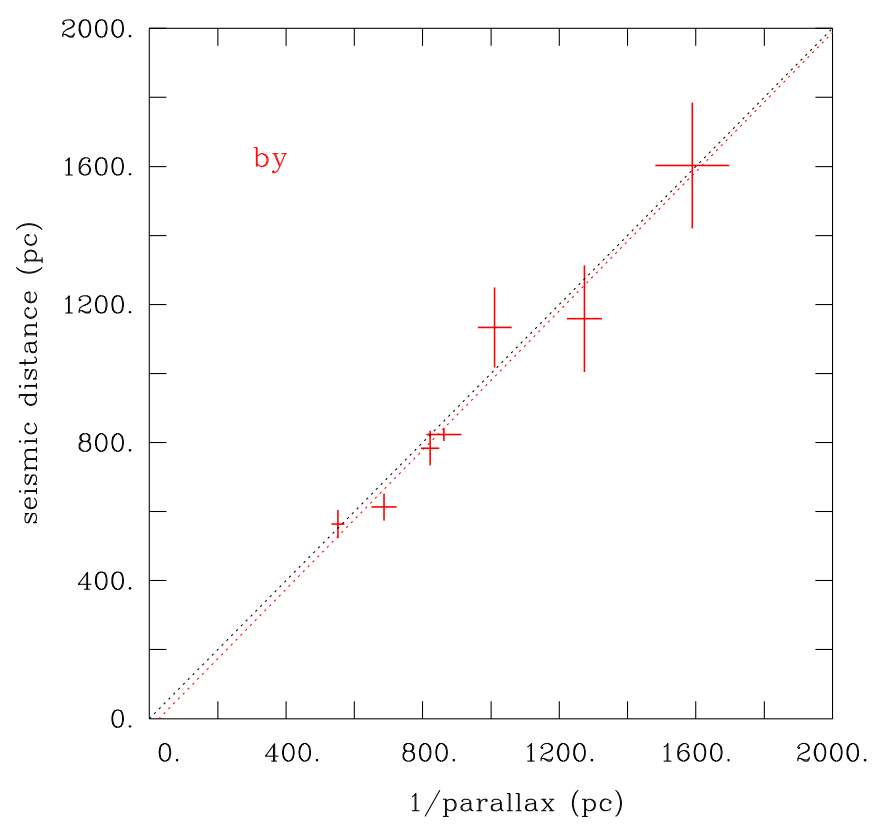

(c)

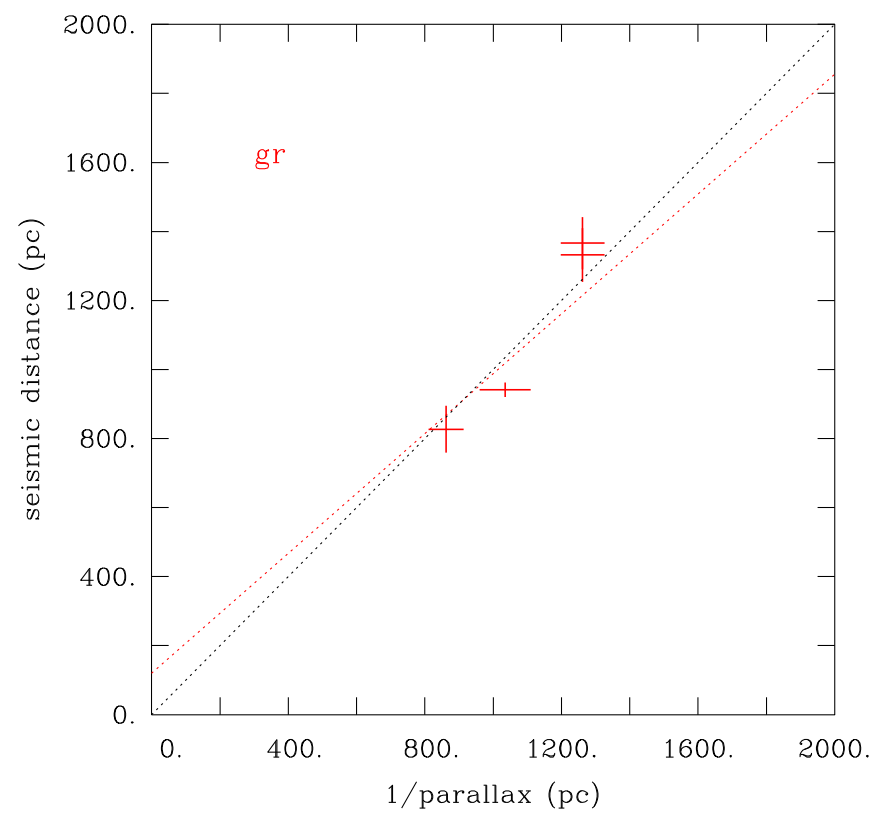

(d)

Figure 3. (Continued.)

other hand, a lower limit can be estimated by assuming maximum interstellar reddening, i.e., the values given by the index $E(B-V)_{\text {mean }}$ in Table 5 . This is now given by $d(\mathrm{~min})$ in Table 6, where we compiled the results for all of the five binary systems in our sample using the available photometry. We also provided the results for the full range of possible values from $d$ (min) to $d(\max )$ including the uncertainties. This range gives a conservative view of seismic distances for those five pulsators. These values clearly overlap with the Gaia distances.

A last point concerns the parallax measurement for PG 0014 +067 . The Gaia uncertainty on that parallax is $37 \%$, well above the values of $1 \%-9 \%$ which characterize the rest of the sample. From the detailed work of Bailer-Jones (2015), it is known that inverting the parallax to obtain a distance in cases where the uncertainty is larger than $\sim 20 \%$ generally leads to significant underestimates of that distance. To investigate this point in the present case, we solved Equation (19) of BailerJones in the context of her exponentially decreasing density galactic model. We adopted the representative exponential scale height of $450 \mathrm{pc}$ as appropriate for sdB stars (Villeneuve et al. 1995). This provides a revised a posteriori estimate of the true distance. Our results are summarized in Table 7, which shows the value of the distance obtained by inverting the parallax (as we have done above) compared to the revised estimate. As expected, the revision leads to negligible changes for all our targets, except for PG $0014+067$ whose distance to 
Table 5

Inferred Interstellar Reddening

\begin{tabular}{|c|c|c|c|c|c|}
\hline Name & $E(B-V)_{\text {mean }}$ & $\begin{array}{l}E(B-V) \\
\left(G_{\mathrm{BP}} G_{\mathrm{RP}}\right)\end{array}$ & $\begin{array}{c}E(B-V) \\
(B V)\end{array}$ & $\begin{array}{c}E(B-V) \\
(\text { by })\end{array}$ & $\begin{array}{c}E(B-V) \\
(g r)\end{array}$ \\
\hline PG $1047+003$ & $0.037 \pm 0.001$ & $0.012 \pm 0.018$ & $0.003 \pm 0.012$ & $0.001 \pm 0.010$ & $\ldots$ \\
\hline PG 0014+067 & $0.033 \pm 0.002$ & $0.155 \pm 0.030$ & $0.114 \pm 0.042$ & $\ldots$ & $0.078 \pm 0.019$ \\
\hline PG $1219+534$ & $0.017 \pm 0.001$ & $0.000 \pm 0.014$ & $\ldots$ & $0.000 \pm 0.028$ & $\ldots$ \\
\hline Feige 48 & $0.018 \pm 0.002$ & $0.046 \pm 0.011$ & $0.031 \pm 0.018$ & $0.015 \pm 0.027$ & $\ldots$ \\
\hline EC 05217-3914 & $0.022 \pm 0.001$ & $0.026 \pm 0.020$ & $0.024 \pm 0.024$ & $0.033 \pm 0.035$ & $\ldots$ \\
\hline PG $1325+102$ & $0.027 \pm 0.001$ & $0.008 \pm 0.008$ & $0.018 \pm 0.049$ & $0.005 \pm 0.003$ & $0.000 \pm 0.036$ \\
\hline PG $0048+091$ & $0.057 \pm 0.001$ & $\ldots$ & $\ldots$ & $\ldots$ & $\ldots$ \\
\hline EC 20117-4014 & $0.051 \pm 0.001$ & $\ldots$ & $\ldots$ & $\ldots$ & $\ldots$ \\
\hline PG 0911+456 & $0.010 \pm 0.001$ & $0.001 \pm 0.009$ & $0.032 \pm 0.037$ & $\ldots$ & $0.001 \pm 0.005$ \\
\hline BAL 090100001 & $0.092 \pm 0.004$ & $0.090 \pm 0.021$ & $0.031 \pm 0.124$ & $\ldots$ & $\ldots$ \\
\hline EC 09582-1137 & $0.057 \pm 0.001$ & $0.068 \pm 0.010$ & $0.057 \pm 0.035$ & $\ldots$ & $\ldots$ \\
\hline KPD $1943+4058$ & $0.212 \pm 0.005$ & $0.124 \pm 0.012$ & $0.134 \pm 0.023$ & $0.147 \pm 0.058$ & $\ldots$ \\
\hline KPD 0629-0016 & $0.869 \pm 0.009$ & $0.300 \pm 0.013$ & $\ldots$ & $0.198 \pm 0.038$ & $\ldots$ \\
\hline KIC $02697388^{a}$ & $0.144 \pm 0.013$ & $0.130 \pm 0.010$ & $0.092 \pm 0.038$ & $\ldots$ & $0.066 \pm 0.007$ \\
\hline KIC $02697388^{b}$ & $0.144 \pm 0.013$ & $0.129 \pm 0.011$ & $0.091 \pm 0.039$ & $\ldots$ & $0.066 \pm 0.008$ \\
\hline PG 1336-018 & $0.046 \pm 0.004$ & $0.085 \pm 0.020$ & $\ldots$ & $0.038 \pm 0.014$ & $\ldots$ \\
\hline TIC 278659026 & $0.024 \pm 0.001$ & $0.004 \pm 0.016$ & $0.006 \pm 0.121$ & $\ldots$ & $\ldots$ \\
\hline
\end{tabular}

Notes.

${ }^{a}$ Seismic Model 1 from Charpinet et al. (2011).

${ }^{\mathrm{b}}$ Seismic Model 2 from Charpinet et al. (2011).

Table 6

Lower and Upper Limits on Seismic Distances for Binary Systems

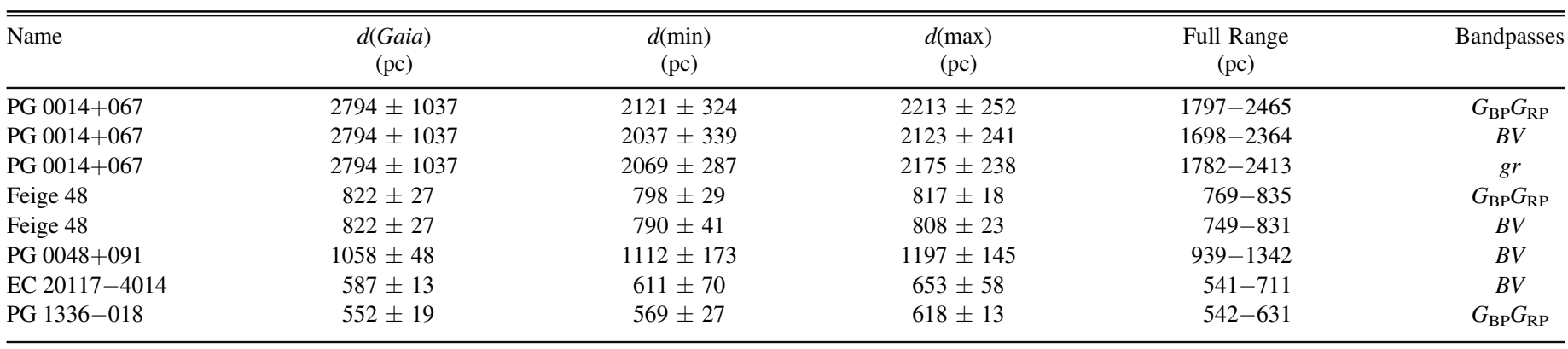

would now be $2156 \mathrm{pc}$ instead of $2794 \mathrm{pc}$, a substantial reduction. Note that $2156 \mathrm{pc}$ is in the middle of the ranges of seismic distance listed in Table 6.

\section{Conclusion}

The recent availability of high-accuracy parallaxes from Gaia DR2 has provided an opportunity to further test the seismic models for pulsating $\mathrm{sdB}$ stars that have been developed over the last several years. The latter are based on the forward modeling approach developed initially by Brassard et al. (2001), which uses parameterized static stellar models. Over the last decade, the method has been refined on the basis of more sophisticated models and optimization methods as described by Charpinet et al. (2016). A total of 16 pulsating sdBs have now been scrutinized through this seismic approach. Of particular interest are the four $g$-mode pulsators for which core probing was achieved. These are KPD 1943+4058, KPD 0629-0016, KIC 02697388, and TIC 278659026 (see Table 1 for the references).
Table 7

Revised Gaia Distances

\begin{tabular}{lcc}
\hline \hline Name & $\begin{array}{c}\text { 1/Parallax } \\
(\mathrm{pc})\end{array}$ & $\begin{array}{c}\text { Posterior Estimate } \\
(\mathrm{pc})\end{array}$ \\
\hline PG 1047+003 & 686.8 & 688.0 \\
PG 0014+067 & 2793.6 & 2156.0 \\
PG 1219+534 & 548.7 & 549.0 \\
Feige 48 & 822.2 & 823.0 \\
EC 05217-3914 & 1590.4 & 1580.0 \\
PG 1325+102 & 861.8 & 863.0 \\
PG 0048+091 & 1058.3 & 1050.0 \\
EC 20117-4014 & 586.9 & 588.0 \\
PG 0911+456 & 1035.0 & 1034.0 \\
BAL 090100001 & 365.6 & 366.0 \\
EC 09582-1137 & 1552.9 & 1536.0 \\
KPD 1943+4058 & 1273.9 & 1273.0 \\
KPD 0629-0016 & 1010.5 & 1010.0 \\
KIC 02697388 & 1261.9 & 1260.0 \\
PG 1336-018 & 552.0 & 553.0 \\
TIC 278659026 & 203.7 & 204.0 \\
\hline
\end{tabular}


The results of our investigation are summarized in Table 4, supplemented by Table 6 for the five binary systems in our sample, where the parallax distance is compared to values based on seismic estimates of the surface gravity, effective temperature, and total mass in conjunction with the available two-band photometry in four different systems. All of the values of the seismic distance are found to overlap with the Gaia distance within $1 \sigma$ uncertainties. In addition, the inferred interstellar reddening is found to be consistent with line-ofsight extinction measurements as provided in Table 5 (taking into account intrinsic reddening in the binaries). This excellent agreement adds further credibility to the seismic models that have been calculated through the parameterized forward modeling approach developed for $\mathrm{sdB}$ stars. The method should find many more applications in the near future as TESS is now providing tens of new light curves for pulsating $\mathrm{sdB}$ stars, among many other outstanding data.

This work was supported in part by the NSERC Canada. G.F. also acknowledges the contribution of the Canada Research Chair Program. S.C. acknowledges financial support from the Centre National d'Études Spatiales (CNES, France) and from the Agence Nationale de la Recherche (ANR, France) under grant ANR-17-CE31-0018, funding the INSIDE project. V.V.G. is a F.R.S.-FNRS Research Associate. M.L. acknowledges funding from the Deutsche Forschungsgemeinschaft (grant DR 281/35-1).

This work has made use of data from the European Space Agency (ESA) mission Gaia (https://www.cosmos.esa.int/ gaia), processed by the Gaia Data Processing and Analysis Consortium (DPAC, https://www.cosmos.esa.int/web/gaia/ dpac/consortium). Funding for the DPAC has been provided by national institutions, in particular the institutions participating in the Gaia Multilateral Agreement. This research has made use of the NASA/ IPAC Infrared Science Archive, which is operated by the Jet Propulsion Laboratory, California Institute of Technology, under contract with the National Aeronautics and Space Administration.

\section{ORCID iDs}

P. Bergeron (1) https://orcid.org/0000-0003-2368-345X

M. Latour (10) https://orcid.org/0000-0002-7547-6180

\section{References}

Alam, S., Alberati, F. D., Allende Prieto, C., et al. 2015, ApJS, 219, 12A, (SDSS DR12)

Allard, F., Wesemael, F., Fontaine, G., Bergeron, P., \& Lamontagne, R. 1994, AJ, 107, 1565

Bailer-Jones, C. 2015, PASP, 127, 994

Baran, A., Pigulski, A., Koziel, D., et al. 2005, MNRAS, 360, 737

Bergeron, P., Saffer, R. A., \& Liebert, J. 1992, ApJ, 394, 228

Bern, K., \& Wramdemark, S. 1973, LowOB, 8, 1

Billères, M., \& Fontaine, G. 2005, in ASP Conf. Ser. 334, 14th European Workshop on White Dwarfs, ed. D. Koester \& S. Moehler (San Francisco, CA: ASP), 635

Brassard, P., Fontaine, G., Billères, M., et al. 2001, ApJ, 563, 1013

Brassard, P., Fontaine, G., Chayer, P., \& Green, E. M. 2010, in AIP Conf. Proc. 1273, 17th European White Dwarf Workshop, ed. K. Werner \& T. Rauch (Melville, NY: AIP), 259

Charpinet, S. 1999, PhD thesis, Univ. de Montréal
Charpinet, S., Brassard, P., Fontaine, G., et al. 2019, A\&A, submitted

Charpinet, S., Fontaine, G., \& Brassard, P. 2003, in NATO ASIB Proc. 105, White Dwarfs, ed. D. de Martino (Dordrecht: Kluwer), 69

Charpinet, S., Fontaine, G., Brassard, P., et al. 2005a, in ASP Conf. Ser. 334, 14th European Workshop on White Dwarfs, ed. D. Koester \& S. Moehler (San Francisco, CA: ASP), 619

Charpinet, S., Fontaine, G., Brassard, P., et al. 2005b, A\&A, 443, 251

Charpinet, S., Fontaine, G., Brassard, P., Chayer, P., \& Green, E. M. 2006a, MmSAI, 77, 358

Charpinet, S., Fontaine, G., Brassard, P., Green, E. M., \& Chayer, P. 2005c A\&A, 437, 575

Charpinet, S., Green, E. M., Baglin, A., et al. 2010, A\&A, 516, L6

Charpinet, S., Silvotti, R., Bonanno, A., et al. 2006b, A\&A, 459, 565

Charpinet, S., Van Grootel, V., Fontaine, G., et al. 2011, A\&A, 530, A3

Charpinet, S., Van Grootel, V., Fontaine, G., et al. 2016, in XXIX IAU General Assembly 1, Astronomy in Focus, ed. P. Benvenuti (Cambridge: Cambridge Univ. Press), 581

Charpinet, S., Van Grootel, V., Reese, D., et al. 2008, A\&A, 489, 377

Fontaine, G., Brassard, P., Charpinet, S., et al. 2003, ApJ, 597, 518

Fontaine, G., Brassard, P., Charpinet, S., et al. 2012, A\&A, 539, A12

Gaia Collaboration, Babusiaux, C., van Leevwen, F., et al. 2018, A\&A, 616, A10, (Gaia DR2)

Green, E. M., Fontaine, G., Hyde, E. A., For, B.-Q., \& Chayer, P. 2008, in ASP Conf. Ser. 392, Hot Subdwarf Stars and Related Objects, ed. U. Heber, S. Jeffery, \& R. Napiwotzki (San Francisco, CA: ASP), 75

Green, E. M., Fontaine, G., Reed., M. D., et al. 2003, ApJL, 583, L31

Heber, U. 2016, PASP, 128, 082001

Hog, E., Fabricius, C., Makarov, V. V., et al. 2000, A\&A, 355, 27

Jester, S., Schneider, D. P., Richards, G. T., et al. 2005, AJ, 130, 873

Kilkenny, D., Koen, C., O'Donoghue, D., \& Stobie, R. S. 1997, MNRAS, 285,640

Kilkenny, D., O’Donoghue, D., Koen, C., Lynas-Gray, A. E., \& van Wyk, F. 1998, MNRAS, 296, 329

Kilkenny, D., Stobie, R. S., O’Donoghue, D., et al. 2006, MNRAS, 367, 1693

Koen, C., O'Donoghue, D., Kilkenny, D., \& Pollaco, D. L. 2004, NewAR, 9,565

Koen, C., O’Donoghue, D., Kilkenny, D., Stobie, R. S., \& Saffer, R. A. 1999, MNRAS, 306, 213

Landolt, A. U. 2009, AJ, 137, 4186

Latour, M., Fontaine, G., \& Green, E. 2014a, in ASP Conf. Ser. 481, 6th Conf. Hot Subdwarf Stars and Related Objects, ed. V. Van Grootel et al. (San Francisco, CA: ASP), 91

Latour, M., Fontaine, G., Green, E. M., Brassard, P., \& Chayer, P. 2014b, ApJ, 788, 65

Németh, P., Kawka, A., \& Vennes, S. 2012, MNRAS, 427, 2180

O'Donoghue, D., Kilkenny, D., Koen, C., et al. 2013, MNRAS, 431, 240

O’Donoghue, D., Lynas-Gray, A. E., Kilkenny, D., Stobie, R. S., \& Koen, C. 1997, MNRAS, 285, 657

Østensen, R. H., Oreiro, R., Solheim, J.-E., et al. 2010, A\&A, 513, A6

Otani, T., Oswalt, T. D., Lynas-Gray, A. E., et al. 2018, ApJ, 859, 1450

O’Toole, S. J., Heber, U., \& Benjamin, R. A. 2004, A\&A, 422, 1053

Randall, S. K., Fontaine, G., Charpinet, S., et al. 2006a, ApJ, 648, 637

Randall, S. K., Fontaine, G., Green, E. M., et al. 2006b, ApJ, 643, 1198

Randall, S. K., Green, E. M., Fontaine, G., et al. 2006c, ApJ, 645, 1464

Randall, S. K., Green, E. M., Van Grootel, V., et al. 2007, A\&A, 476, 1317

Randall, S. K., Van Grootel, V., Fontaine, G., Charpinet, S., \& Brassard, P. 2009, A\&A, 507, 911

Reed, M. D., Telting, J. H., Ketzer, L., et al. 2019, MNRAS, 483, 2282

Schlafly, E. F., \& Finkbeiner, D. P. 2011, ApJ, 737, 103

Schuh, S., Huber, J., Dreizler, S., et al. 2006, A\&A, 445, 31

Van Grootel, V. 2008, PhD thesis, Univ. de Toulouse

Van Grootel, V., Charpinet, S., Brassard, P., Fontaine, G., \& Green, E. M. 2013, A\&A, 553, A97

Van Grootel, V., Charpinet, S., Fontaine, G., et al. 2008, A\&A, 488, 685

Van Grootel, V., Charpinet, S., Fontaine, G., et al. 2010a, ApJL, 718, L97

Van Grootel, V., Charpinet, S., Fontaine, G., Green, E. M., \& Brassard, P. 2010b, A\&A, 524, 63

Villeneuve, B., Wesemael, F., Fontaine, G., Carignan, C., \& Green, R. F. 1995, ApJ, 446, 646

Wesemael, F., Fontaine, G., Bergeron, P., Lamontagne, R., \& Green, R. F. 1992, AJ, 104, 203

Zacharias, N., Finch, C. T., Girard, T. M., et al. 2013, AJ, 145, 44 